\title{
Los acentos del miedo: la construcción biopolítica de lo extranjero en el cuento "Un regalo" de Yolanda Oreamuno
}

Por: Dr. Carlos M. Villalobos Villalobos ${ }^{1}$, Universidad de Costa Rica

Fecha de recibido: $\quad 6$ de marzo, 2016.

Fecha de aceptación: 25 de agosto, 2016.

\section{Resumen}

Con base en el concepto foucaultiano de biopolítica, este artículo analiza el cuento "Un regalo" de Yolanda Oremuno. En este relato se presenta la historia de un enfrentamiento no verbal entre un sujeto nacional y un extranjero. Desde la semiótica del miedo, se analiza cómo se construye esta mirada del prejuicio. El cuerpo del extranjero es representado como un monstruo raro, como un riesgo de contagio frente al asombro y rechazo del local. Finalmente, se prueba que en esta dinámica se genera la paradoja del biopoder, pues el cuerpo que rechaza es al final de cuentas el que más padece lo monstruoso.

\section{Abstract}

The accents of fear: the biopolitical construction of the foreign in the short story "Un regalo" by Yolanda Oreamuno

Based on a Fouculian concept of biopolitics, this paper analyzes the short story "Un regalo" by Yolanda Oremuno. This narration presents the story of a non-verbal confrontation between the national individual and a foreigner. From the semiotics of fear, how this prejudice is constructed is thus analyzed. The body of the foreigner is represented by a bizarre monster, as a risk of contagion before the amazement and rejection of the national. Finally, it is proven that in this dynamics a biopower paradox is generated, since the rejection body is the one that suffers the most in the face of monstrosity.

1 El doctor Carlos Manuel Villlobos es profesor Catedrático de Teoría Literaria y Semiótica en la Universidad de Costa Rica. Es doctor en Letras y Artes en Centro América, máster en Literatura Latinoamericana y licenciado en Periodismo. Contacto: carlos.villalobos@ucr. ac.cr. 
Al que está solo le crecen murallas por enfrente; se le agrandan los seres humanos hasta monstruos; se le confunden con los árboles, los gatos y las puertas.

Yolanda Oreamuno

Una de las vetas de la investigación cultural que recientemente ha llamado la atención de las comunidades académicas, es la relación existente entre el poder, la cotidianidad y las representaciones del cuerpo. Este interés, que parte de la propuesta esbozada por Michel Foucault sobre vida y poder o biopolítica, ha propiciado diversos estudios que intentan dar cuenta de las marcas semióticas del cuerpo relacionadas con temas tales como la nutrición, la enfermedad, los códigos de la vestimenta, las intervenciones físicas o las configuraciones corporales $y$, a la vez, cómo funciona el anclaje político de estas representaciones.

La biopolítica, también conocida en algunos contextos como biopoder, es la disciplina que estudia cómo infieren los poderes sociales sobre las regulaciones corporales de los ciudadanos. De acuerdo con Michael Foucault (2012), "La biopolítica tiene que ver con la población, y ésta como problema a la vez científico y político, como problema biológico y problema de poder (...). La biopolítica abordará, en suma, los acontecimientos aleatorios que se producen en una población tomada en su duración" (p. 222). Para el teórico social alemán Thomas Lemke (2011) la noción de biopolítica se refiere al surgimiento de un conocimiento político específico y una nueva disciplina que relaciona estadística, demografía, epidemiología y biología. Según este autor, "These disciplines make it possible to analyze processes of life on the level of populations and to "govern" individuals and collectives by practices of correction, exclusion, normalization, disciplining, therapeutics, and optimization"2 (p. 5).

En este contexto el concepto de "población", juega un papel autónomo ambiguo, pues representa una

2 Estas disciplinas permiten analizar procesos de vida a nivel de poblaciones y "gobernar" a individuos y colectividades mediante prácticas de corrección, exclusión, normalización, disciplina, terapéutica y optimización (Traducción propia). realidad colectiva que no necesariamente depende de la intervención política, sino de su propia dinámica y modos de autorregulación. Esta autonomía, sin embargo, no es capaz de filtrar todas las intervenciones del poder (político, económico, religioso, etc.). En esta dinámica, el cuerpo funciona como representación de la colectividad y, por lo tanto, es un soporte semiótico plurisignificativo, pues reproduce y, al mismo, tiempo rechaza las dinámicas simbólicas de la población y los poderes que la regulan. En el juego de estas aporías, las corporalidades son un universo fascinante, una metonimia viviente, que inscribe dinámicas sociales heterogéneas en la piel de cada cuerpo.

Si bien el concepto de biopolítica no es exclusivamente de Foucault, se le debe a este filósofo francés la principal aportación conceptual. De acuerdo con Lemke (2011), es posible diferenciar tres maneras distintas de emplear esta noción:

First, biopolitics stands for a historical rupture in political thinking and practice that is characterized by a rearticulation of sovereign power. Second, Foucault assigns to biopolitical mechanisms a central role in the rise of modern racism. A third meaning of the concept refers to a distinctive art of government that historically emerges with liberal forms of social regulation and individual self-governance. But it is not only the semantic displacements that are confusing. Foucault not only employs the term "biopolitics"; he also sometimes uses the word "biopower," without neatly distinguishing the two notions ${ }^{3}$ (p. 34).

El costarricense Diego Soto (2015), quien ha hecho un amplio análisis de este concepto en la obra de Foucault, plantea que la relación entre vida y política se establece mediante el vínculo sujeto/objeto.

3 En primer lugar, la biopolítica representa una ruptura histórica en el pensamiento y la práctica política, y se caracteriza por una rearticulación del poder soberano. En segundo lugar, Foucault (2000) asigna a los mecanismos biopolíticos un papel central en el surgimiento del racismo moderno. Un tercer significado del concepto se refiere a un arte distintivo del gobierno que emerge históricamente con formas liberales de regulación social y autogobierno individual. Pero no solo los desplazamientos semánticos son confusos. Foucault no sólo emplea el término "biopolítica"; también utiliza a veces la palabra "biopoder", sin distinguir claramente las dos nociones (Traducción propia). 
La vida asume el rol de un objeto particular sobre el cual se aplica el poder político. En el proceso, la condición vital se transforma, se limita, es administrada y no puede decidir sobre sus horizontes de posibilidad. En cambio, "la política se mantiene cerrada sobre sí misma" (p. 176).

Desde esta perspectiva, el sujeto viviente, desde que nace, experimenta múltiples intervenciones: reglas de vestimenta, recatos de intimidad, modelizaciones gestuales o proxémicas, intervenciones estéticas, auscultaciones profilácticas, y más aún: violaciones, sojuzgamientos, mutilaciones, etc. Pero el cuerpo, en esta dinámica social, no solamente es intervenido por reglas pragmáticas cotidianas de codificación estética o de roles específicos; también implica la posibilidad de ser intervenido como objeto de consumo, como target panóptico en los discursos de la seducción publicitaria o pornográfica.

Al otro lado de esta exposición está el cuerpo negado: el obeso, el deformado, el moribundo, el mutilado; en fin, el cuerpo como monstruo. Esta intervención del poder político obliga a los cuerpos que transitan por el espacio a ajustarse a ciertos interdictos. Un cuerpo puede ser, en sí mismo un insulto a los demás. Algunos responden a normas jurídicas asociadas al tema de lo moral, pero otros son rechazos ideológicos o meras semióticas del miedo (prejuicios, estereotipos, xenofobias, homofobias...) En este caso, por ejemplo, un cuerpo totalmente desnudo podría ser tan amenazante como el de una musulmana que se niega a desvestirse en una playa de Francia.

Desde la perspectiva semiótica abordada en este análisis, se enfatizará en el cuerpo como signo de miedo; es decir, como construcción de los estereotipos y las acciones de exclusión. En el marco de las oleadas migratorias, que recientemente se han incrementado, y los discursos xenofóbicos, incluso enunciados abiertamente por poderosos líderes políticos, el cuerpo ajeno es motivo de preocupación, de amenaza y, por lo tanto, de rechazo. La semiótica de este miedo cobra víctimas extranjeras acosadas en las calles o rechazados ad portas en aeropuertos, tan solo porque sus códigos de vestimenta y referencia nacional representan una determinada posición ideológica o religiosa.
Un texto que hace referencia de manera directa a esta semiótica del miedo, vinculada con la presencia corporal del otro como extranjero, es el relato corto "Un regalo" de la escritora costarricense Yolanda Oreamuno. El cuento fue publicado en Repertorio Americano en 1948 y describe una situación extrema experimentada por un extranjero frente a la incomprensión de los habitantes del vecindario a donde ha venido a residir. Este foráneo vive una situación de inadaptación social, pues no logra comprender la lengua y los códigos comunicativos locales. Esta situación se agrava pues es objeto de un prejuicio comunal que lo rechaza, al punto de actuar, frente a él, con absoluta indiferencia.

Un antecedente posible de este relato de Oreamuno se encuentra en el texto El extranjero (1942) del argelino-francés, Albert Camus. El texto retrata la pérdida de la empatía social. El protagonista ni siquiera se inmuta ante la muerte de su madre $y$, como parte de su proceso degradación, termina convertido en un asesino. Esta visión pesimista se inscribe en el marco de las secuelas que dejaron las dos guerras mundiales, no solo en cuanto al impacto psicológico, sino en relación con la caída de los imaginarios utópicos. Nada parecía tener sentido entonces. El extranjero es la imagen de la desilusión, la pérdida total de la fe en los principios del iusnaturalismo. La razón y la ética se pierden totalmente; y el mundo, ahora sin máscaras evidencia, tal cual, la mezquindad y la maldad acérrima de la naturaleza humana.

El personaje camusiano replantea el sentido etimológico de "extranjero", pues aquí no es exactamente un foráneo geográfico; sino alguien que no logra integrarse. Es un personaje psicópata ubicado en un lugar de distanciamiento social. En este caso también se crean las barreras que separan a los unos y a los otros y, en ambos casos, los cuerpos presentan dinámicas semióticas de miedo y de rechazo. Por esta misma razón, al final de la narración el protagonista es sentenciado a muerte, es decir, es expulsado o expurgado como la representación de lo extraño para la colectividad heterodoxa.

En el texto de Yolanda Oreamuno, el extranjero reproduce algunos de estos rasgos camusianos, pues también el personaje es un ser extraño generador de asombro y malestar en los locales. En resumen, es la 
representación de la diferencia. Sin embargo, en el cuento de Oreamuno, este extranjero no manifiesta conductas tan antisociales como en Camus, a pesar de que también en este caso el extranjero es, virtualmente, asesinado por la comunidad que lo rechaza. Por otra parte, en "Un regalo" (1961), la patología más evidente la manifiesta el personaje local que espía al extranjero, pues se describe como un resentido social, como un sujeto solo que es incapaz de socializar. En consecuencia, desde la perspectiva del nativo, el extranjero es el anormal, el monstruo; pero en la propuesta de Oreamuno la mayor anomalía se aplica a la actitud de indiferencia y nula capacidad de compasión característica del protagonista. De este modo, la escritora rompe con el imaginario camusiano, pues evidencia que la mirada del local es la que construye las representaciones simbólicas de acusación, miedo y exclusión. La mayor monstruosidad no la carga el foráneo, sino el ojo ciego de quien le atribuye esta condición.

A pesar de que el sujeto local comparte con el extranjero la soledad y el resentimiento, no es capaz de intentar una comunicación con él. El prejuicio con el que lo mira, lo lleva a esquivarlo y le atribuye rasgos animalescos para describirlo: "Caminaba cayendo. Sus pasos no eran pasos, eran botes, saltos, sentones. Como canguro. Y esa reminiscencia animal, tampoco canalizaba lo inanimado de su figura" (p. 178).

Esta representación zoomórfica, como construcción de lo monstruoso, activa una alerta de peligro; por lo tanto, una instauración del miedo como mecanismo de respuesta natural. El cuerpo da susto y asco: es un cuerpo trastocado por la mirada. Como consecuencia, el extraño es objeto del estereotipo que impide conocer la especificidad humana y atribuye rasgos negativos a la generalidad. Detrás de esta representación es posible encontrar mecanismos ideológicos que configuran un esquema biopolítico, en el cual lo local es el espacio de seguridad y la presencia del otro, el de la inseguridad. Una de las fuentes de este biopoder lo constituye la idea de lo nacional, como representación de una identidad homogénea y estable; es decir, que no admite el riesgo de la desestabilización semiótica.
Por esta razón, en este caso, el vecino se dedica, con sigilo, a vigilar al foráneo como se observa en la siguiente cita, en la cual además, le atribuye rasgos físicos antiestéticos:

Concordancia en horas de salida y entrada; vecindad de puertas; uniformidad de pobrezas, familiar obsesión de estarlo mirando, le hizo casi llegar a tenerle miedo. Simbolizaba alguna raza caída, sin patria ni recuerdo. Cuando se encontraban frente a frente, se quedaba fijo con una lágrima seca y un implorante deseo de algo en la pupila. Al caminar por el pasillo se acercaba como para tocarle. Daba espanto. Un poco de lástima y un poco de asco. Todo desagradable. Todo feo. Sintió también que el hombre olía a pescado, sin que esa reminiscencia marina abriera el horizonte de angustia que sugería (p. 178).

En esta cita se evidencia la danza no verbal de ambos cuerpos. En esta esfera, el biopoder pertenece al nativo. Desde este lugar, el cuerpo del extranjero representa la invasión del espacio personal. Este traspaso de la frontera personal genera una disconformidad primitiva. Se trata de la activación de la defensa territorial que funciona en este tipo de situaciones como arquetipo de base. En la estructura superficial las marcas del arquetipo se manifiesta como incomodidad irracional, como asombro y como temor. Por eso el cuerpo del otro es representado como espantoso, desagradable, feo y maloliente.

El extranjero, como sujeto perteneciente a otro territorio, más allá de ser un concepto jurídico construido principalmente por la lógica de formaciones nacionales, se sitúa, semánticamente en contraposición a los sujetos que sienten una determinada pertenencia territorial, incluso aquellos que han logrado adaptarse y adoptar esta identificación territorial por naturalización o nacionalización. En principio, lo extranjero supone que alguien ha traspasado la delimitación simbólica de la frontera divisoria entre lo propio y lo ajeno. Este individuo, con sus propios códigos culturales, puede producir, sobre todo en contextos socialmente más cerrados, reacciones de rechazo y convertirse en un amenazante para la comunidad local, o al menos para una parte de esta. 
De acuerdo con Carlos Sandoval García, "La dimensión subjetiva de las identidades nacionales es frecuentemente representada como la preservación de las fronteras nacionales, como la protección de una personalidad colectiva. Hay íconos que consideran un "hogar" como un lugar de seguridad" (2002, p. $11)$.

En esta dinámica se reproduce la mayor ambigüedad en los procesos de intervención del cuerpo como semiótica de la otredad, pues la mirada que lo ausculta desde la curiosidad, en un primer momento, puede sentir atracción o asombro, pero en cuanto tiene conciencia de la diferencia, reacciona con miedo y con desprecio. Es decir, el cuerpo del otro es un lugar sensible para el biopoder que defiende el statu quo de lo nacional. Así, quien pasa la frontera, sin más explicación, puede resultar un amenazante.

Siguiendo con los planteamientos de Carlos Sandoval, "las naciones son consideradas no tanto como "comunidades" sino como formaciones de diferencia y desigualdad. Esta compleja interrelación de permanencia y diferencia puede ser explotada a través de tres dimensiones cruciales: formación de subjetividades, representaciones y factores materiales" (p. 8). Las subjetividades se enmarcan en las dinámicas de la psicología, las representaciones configuran los códigos de las identidades y los factores materiales explican los condicionantes sociales de las movilizaciones. "Este tipo de constitución de la subjetividad genera una conflictiva fusión de poder, temor, ansiedad y deseo" (p. 11).

En el cuento "Un regalo", entre estas dimensiones cruciales destaca la que hace referencia a la subjetividad, pues es la que activa el miedo del protagonista e incide directamente en la representación del otro como una amenaza. Es aquí donde ocurre con más fuerza la construcción del estereotipo.

Véase la manera cómo continúa la descripción hecha por el protagonista del cuento y la idea de que el otro es un ser extraño: "Las terribles vibraciones se iban compactando en la garganta angustiada de aquel ser raro: agrupando como ovejitas vencidas; trenzando como nudo o sollozo, se iban haciendo más y más roncas, más y más lentas, más y más cargadas" (p. 182). Esta cita evidencia cómo opera en el discurso, la semiótica del miedo construida a partir de la violación arquetípica de la seguridad territorial.

Ruth Cubillo, en su análisis de algunos de los cuentos de Yolanda Oreamuno, considera que estos podrían explicarse con base en los postulados freudianos esbozados en el conocido ensayo sobre El malestar en la cultura. En los relatos oreamunianos se evidencian las tres fuentes principales de las acciones del displacer según Freud: el miedo a la muerte, el miedo a la amenaza del mundo exterior (naturaleza) y el temor a las relaciones sociales. A propósito del cuento "Un regalo", Cubillo (2011) plantea que el narrador "agrede de múltiples formas al solitario: con la evasión, la negación de compañía y la negación de un poco de pastel" (p. 146).

La indiferencia mostrada por el protagonista del relato incide, indirectamente, en la muerte del extranjero, pues evidentemente si hubiera habido alguna manifestación de solidaridad en el día del festín, no se produce su muerte. La motivación de fondo que propicia esta negativa de ayuda, se justifica, como ya se ha apuntado, por el temor producido en el sujeto por la amenaza del otro y por la idea de poner en riesgo su espacio de seguridad. La paradoja aquí es que esta amenaza es solo un prejuicio y, más bien, quien resulta la única víctima de esta actitud es el extranjero, quien finalmente paga con su propio deceso.

Otra de las configuraciones de la subjetividad asociadas al miedo en relación con el otro, es la representación del peligro de contagio. De acuerdo con Mary Douglas (1966), hay cuatro formas básicas de representación del miedo a ser contaminados: "The first is danger pressing on external bouderies; the second danger from transgressing the internal lines of the system; the third, danger in the margins of the lines. The fourth is danger from internal contradiction $^{4}$. (pp. 123-124).

En el cuento "Un regalo", el extranjero resulta un amenazante para la salud propia, pues ha cruzado estas líneas simbólicas. El temor a ser contagiados es la principal motivación del distanciamiento y rechazo.

$4 \quad$ La primera es peligro de presión sobre las fronteras externas; la segunda, peligro de transgredir las líneas internas del sistema; la tercera, peligro en los márgenes de las líneas. El cuarto es peligro de la contradicción interna (traducción propia). 
El cuerpo del otro podría contener elementos patógenos de alto riesgo y, dado que no es posible aplicar aquí el antiguo mecanismo de la cuarentena, no hay una certeza que garantice la asepsia.

Desde un posible encuadre ético o racional, el protagonista presenta algunos atisbos de conciencia. En primer lugar, es capaz de mirarse a sí mismo y tener conciencia de su comportamiento: "Él debía tener frente al extranjero, la cara embrutecida de estupor y los ojos dilatados de espanto" (p. 182). Pero también es consciente de sus propias contradicciones y reflexiona al respecto: "Él estaba hecho de una angelical tolerancia, unida a una diabólica intransigencia" (p. 183). Sabe que debería ser piadoso, pero no puede: "¿Piedad? ¿Se puede sentir piedad cuando se tiene tanto asombro?" (p. 184).

De acuerdo con Sandoval (2002), no se trata de dar voz a los que no la tienen sino de crear posibilidades de escucha. "La escucha comporta dimensiones analíticas y éticas. Desde la perspectiva analítica requiere disposición para intentar comprender el horizonte de significación de la persona que habla y ello no se logra con una simple conversación" (p. 380). En el caso de este texto, el extranjero intenta comunicarse con su vecino de diversos modos, pero este le niega toda posibilidad de diálogo y, más aún, el acento de la lengua incomprendida le parece que suena a piedra. Las palabras "salían de sus labios disparatadas, y rebotaban con chasquido de leña quemada. Las consonantes hacían virutas de sonido, perseguidas por unas pocas vocales que no lograban darles alcance" (p. 181). Según Sandoval la escucha del otro plantea un desafío ético que supone, en primer lugar, una relación de respeto (p. 380). Esta condición "humanista" o meramente ética no se cumple en esta historia. En medio de la soledad vivida por ambos personajes, se instala un muro imposible de traspasar: de un lado, el desconocimiento de la lengua local imposibilita la comunicación; y del otro, la actitud sorda o de rechazo del vecino impide que se dé al menos una rendija para el diálogo.

Como se indicó arriba, en estos atisbos éticos, en efecto, el protagonista comprende que debería tener una actitud "más humana" frente a la desgracia ajena. "Él haría un esfuerzo; o dos esfuerzos, o tres, si tantos eran necesarios para acercarse o, mejor di- cho, para dejarse aproximar de un ser tan desprovisto de atractivos. Él lo haría. O esperaría que el otro lo hiciese" (p. 179). Pero el personaje carga un resentimiento que le impide la comunicación y la solidaridad, a pesar de que él también es un hombre solo: "Pero allí, en ese rincón de sí mismo donde el hombre existe para su propio regocijo o dolor, él también estaba solo" (p. 183).

El extranjero toca el timbre para pedirle auxilio a su vecino. Pero ese día, alguien le ha traído un pastel y no hay disposición para compartir. En consecuencia, no le abre la puerta. La contradicción es profunda. La conciencia del personaje tiene el deseo de hacer algo: "Pero tienes el impulso. El impuso esencial de la generosidad, que es lo que importa. Algo conmovedor, "o conmovible" que nace con el invierno y la Navidad. Todo el mundo tiene deseos de ser bueno. La filantropía se pone de moda" (p. 190). Sin embargo, la respuesta final es más bien misántropa. Sin esta ayuda vital, el extranjero morirá de hambre.

Este final pone de manifiesto la incapacidad humana para mirar su propia monstruosidad; es decir, el sujeto enfermo no es el que invadió el lugar del otro, sino quien niega la posibilidad de la estadía, pues el recién llegado es un peligro. De este modo, en el relato, mientras el personaje local vomita de indigestión, el extranjero muere de hambre sin recibir la mínima consideración. Son las dos caras de un mismo malestar. Son las paradojas biopolíticas de una sociedad cuyos factores materiales e institucionales provocan exclusiones.

En la escena final del relato, se contrapone la diferenciación entre ambos personajes, mientras uno acaba de morir, el otro experimenta una sensación de asco en relación con su propia realidad:

Recordó con ira sus literarias palabras de la noche anterior: "El encanto de los pasteles está en el hartazgo, y posteriormente, en la indigestión". ¡Qué desagradable! Se levantó para averiguar qué cosa ocasionaba tanto alboroto; al pasar por el comedor miró los restos del dulce sobre la mesa: un trozo todavía entero y un dorado hormiguero de migajones. Sintió vómito (p. 194). 
El cuento contrapone dos cuerpos intervenidos: uno es objeto de una ingesta desborda, la orgía opípara que conduce al hartazgo, al consumo desmedido y, finalmente, el vómito; el otro es el excluido por su condición estereotipada de extraño, de hambriento que pide sin que le oigan; es el sujeto de la carencia extrema, el otro que, finalmente, es condenado a muerte.

El contexto en el que se desarrolla este cuento es en la ciudad de México a finales de la primera mitad del siglo XX. Aquí Yolanda Oreamuno era entonces extranjera. Aunque podría compartir algunos de los rasgos de estos personajes, como la soledad o el resentimiento, el cuento en principio no es de autoficción. Sin embargo, con "Un regalo", Oreamuno (año de publicación) nos regala una ventana para mirar la indiferencia humana a la cual deben enfrentarse las personas que traspasan las fronteras e ingresan al territorio de "los otros".

Esta es también una ventana para mirarnos a nosotros mismos frente a los nómadas modernos (los inmigrantes del mundo que ahora transitan sus exclusiones por Centroamérica y México; los mojados, los expatriados, los ilegales, los exiliados, los refugiados). Es la historia de los cuerpos en éxodo: sujetos errantes que tropiezan con muros, policías de migración, deportaciones y biopolíticas que responden al arquetipo territorial vigente. Estos sujetos al cruzar las fronteras son amenazantes que vienen a exhibir sus cuerpos carentes y portadores de todo riesgo para los internos. La reacción, como en este cuento de Oreamuno, consistirá en aplicar una táctica posible para exorcizar estos cuerpos endemo- niados. Pero en este lado, la cara más monstruosa es la del perro guardián que ladra furioso en las cercas que protegen nuestro miedo.

\section{REFERENCIAS BIBLIOGRÁFICAS}

Cubillo, R. (2011). Mujeres ensayistas e intelectuales de vanguardia en la Costa Rica de la primera mitad del siglo XX. San José: Editorial Costa Rica.

Douglas, M. (1966). Purtity and danger and analysis of the concepts of pollution and taboo. Londres: Routledge.

Foucault, M. (2000). Defender la Sociedad: Curso en el Collège de France (1975-1976). Traducción al castellano de Horacio Pons. Buenos Aires, Fondo de Cultura Económica.

Foucault, M. (2012). Nacimiento de la biopolítica. Curso en el Collège de France (1978-1979), traducido por Horacio Pons, Buenos Aires: FCE.

Lemke, T. (2011). Biopolitic: An advanced introduction. Traducido por Eric Frederick, New York: New York University Press,

Oreamuno, Y. (1961). A lo largo del corto camino. San José: Editorial Costa Rica.

Sandoval García, C. (2002). Otros amenazantes. Los nicaragüenses y la formación de identidades nacionales en Costa Rica. San José: Editorial UCR.

Soto Morera, D. A. (2015). En carne propia: religión y biopoder. Una lectura de Michel Foucault. San José: Editorial Arlequín. 